\title{
Field Evaluation of GNSS/GPS Based RTK, RTN, and RTX Correction Systems
} US Army Corps of Engineers ${ }_{\circledast}$

by J. Robert Mitchell and Michael F. Forte

PURPOSE: This Coastal and Hydraulic Engineering Technical Note (CHETN) details an evaluation of three Global Navigation Satellite System (GNSS)/Global Positioning System (GPS) real-time correction methods capable of providing centimeter-level positioning. Internetand satellite-delivered correction systems, Real Time Network (RTN) and Real Time eXtended (RTX), respectively, are compared to a traditional ground-based two-way radio transmission correction system, generally referred to as Local RTK, or simply RTK ${ }^{1}$. Results from this study will provide prospective users background information on each of these positioning systems and comparisons of their respective accuracies during in field operations.

\section{BACKGROUND}

Field Research Facility (FRF) Survey Program and Mission. As part of the US Army Engineer Research and Development Center, Coastal and Hydraulics Laboratory, the FRF has strived to fulfill a critical mission of collecting beach/nearshore topographic and bathymetric survey profile data since 1974. Technology and equipment used to collect survey data have progressed through the years. Beginning with the use of an automatic level/stadia rod/survey tape, followed by the incorporation of a Motorola Mini-Ranger, then using electronic theodolites and infrared distance meters (Zeiss Elta-2s, Geotronics Geodimeter 140T) until 1996 when RTK GPS became the operational approach used for survey data collection (Birkemeier and Forte 2019). The GPS/GNSS-based survey data also provide ground control for the testing and operation of remote sensing technologies, and the use of GNSS data is integral during gauge deployment and recovery operations. The facility has a permanent local base station set up where GPS/GNSS signals are received, corrected, and transmitted by very high frequency (VHF) radio in real time to the rover system used during field operations (Forte et al. 2017).

Correction Systems Evaluated. RTK, RTN, and RTX were evaluated at the FRF to compare their respective positioning accuracies during field operations. Of the three positioning systems being evaluated, Local RTK requires the most hardware/equipment. As general operating principles, the Local RTK system must have at least one base-station set up on a very accurately known location. It can then correct the positioning information it receives from the satellites for its location and send the correction data packets to the rover system through VHF radio transmission (Carballido et al. 2014). RTN, also known as Virtual Reference Station (VRS) and Network RTK, is a system consisting of a network of base stations controlled by a government or private organization. The base stations within a given RTN are spread out spatially to allow coverage of a specified region, and the continuous observations are combined to derive a model of the region. From this model, correction data are computed for areas within the region and broadcast from an internet based server to the internet connected rover (Xiaoming et al. 2011). The RTN system may be advantageous over the classic RTK local base and rover system as it decreases the amount of equipment needed by the user in RTK positioning signals

\footnotetext{
${ }^{1}$ Real Time Kinematic
} 
applications. The third positioning system evaluated is a Trimble-owned service called Real Time eXtended (RTX). This system requires the least amount of equipment needed by the user to acquire positioning. It consists of a global network of reference stations that transmit data to RTX server centers where the correction data are computed and global and/or regional atmospheric models are determined and applied (Brandl et al. 2019). For published accuracies of GNSS/GPS positioning methods, see Table 1.

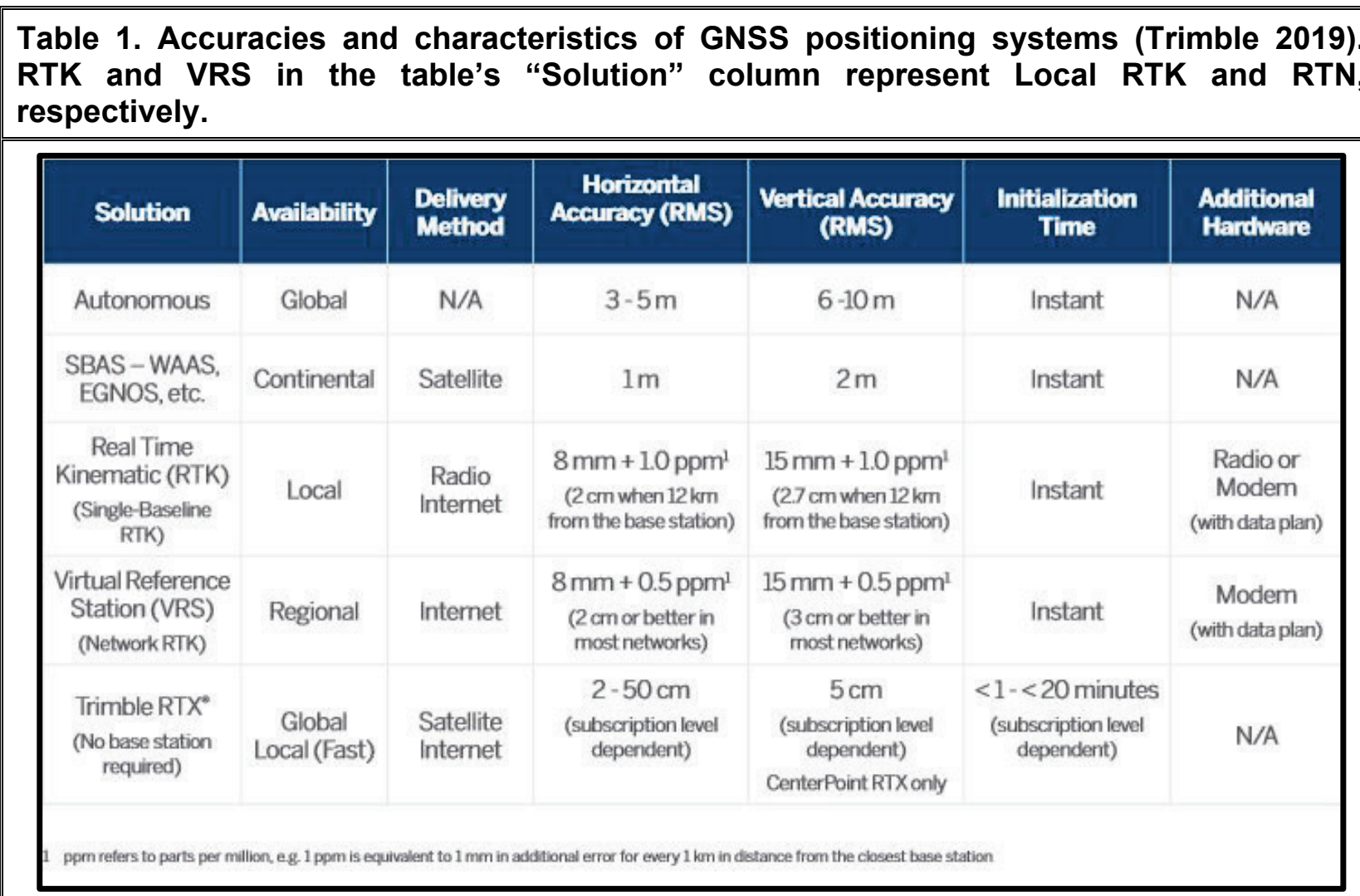

SITE DESCRIPTION AND EQUIPMENT: The field test was conducted in northeastern North Carolina along the northern portion of the Outer Banks and spanned the towns of Corolla, Duck, and Kitty Hawk (Figure 1). The FRF is located in the town of Duck and is bounded by the Atlantic Ocean to the east and Currituck Sound to the west.

Table 2 lists the major components for each system used during the evaluation and includes a list of components shared by all three systems while testing. 


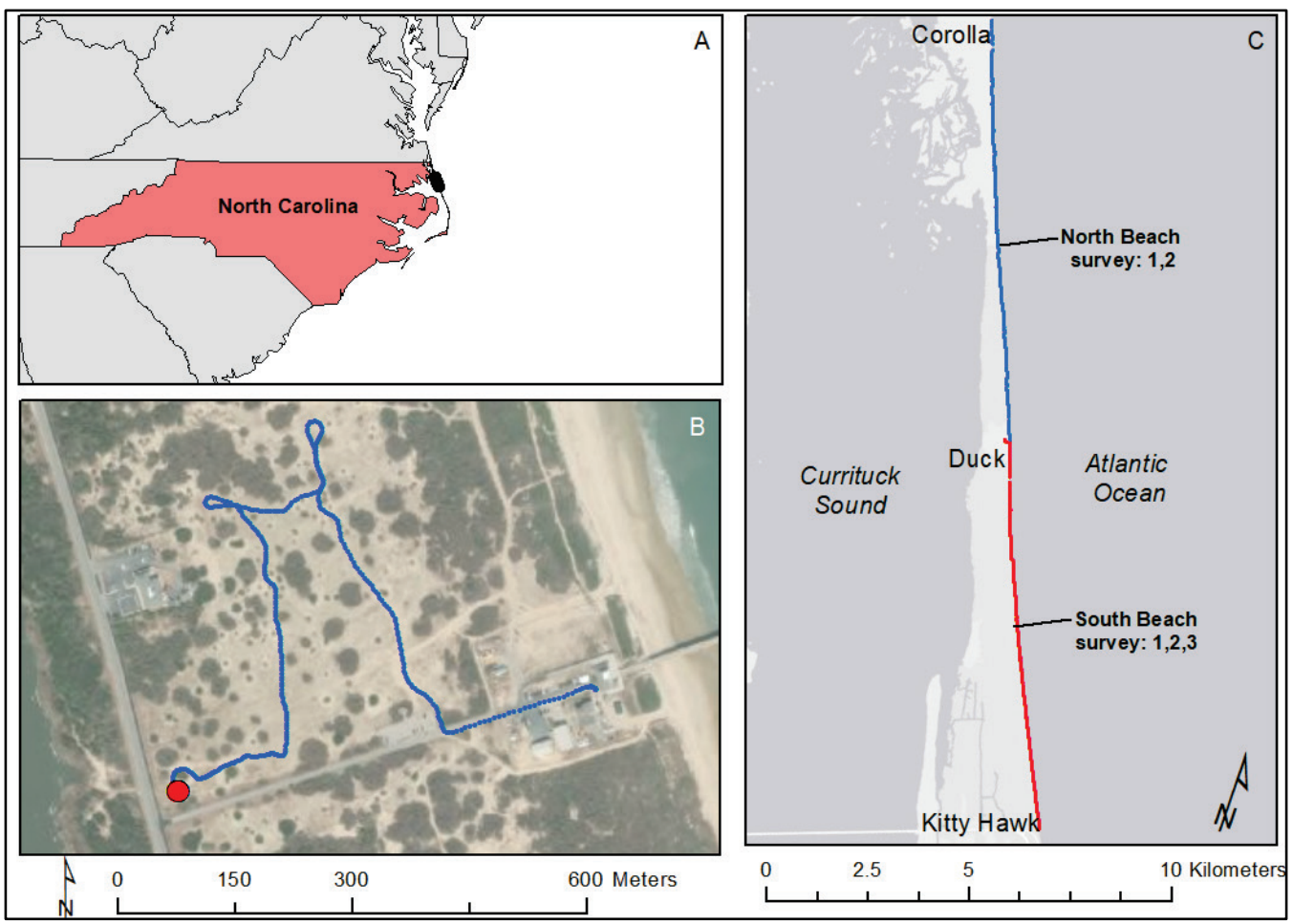

Figure 1. Panel A: Overview map showing North Carolina; black oval represents general location of tests. Panel B: Local overhead view of FRF property; blue dotted line represents path of Dune survey session; red circle represents location of the three static survey sessions. Panel C: Regional view showing extent of surveys; north and south dynamic tests are shown by blue and red lines.

Table 2. Equipment used during the field tests.

\begin{tabular}{|l|l|l|l||}
\hline \multicolumn{4}{|c|}{ Equipment } \\
\hline \multicolumn{1}{|c|}{ Local RTK } & \multicolumn{1}{|c|}{ RTN } & \multicolumn{1}{|c|}{ RTX } & \multicolumn{1}{|c|}{ Shared Equipment } \\
\hline (2) Trimble R7 GNSS receivers & (1) Trimble R7 GNSS receiver & (1) Trimble R9S receiver & $\begin{array}{l}\text { Trimble Zephyr 3 Rover } \\
\text { antenna }\end{array}$ \\
\hline (1) Trimble Geodetic antenna & Verizon MiFi (wireless internet) & $\begin{array}{l}\text { Trimble CenterPoint RTX } \\
\text { subscription }\end{array}$ & $\begin{array}{l}\text { RG-58 GNSS antenna } \\
\text { cable }\end{array}$ \\
\hline RG-58 GNSS antenna cable & $\begin{array}{l}\text { North Carolina Geodetic Survey } \\
\text { (NCGS) RTN subscription }\end{array}$ & $\begin{array}{l}\text { Data cables: reciever- } \\
\text { computer }\end{array}$ & $\begin{array}{l}\text { Instock GPS320 3-way } \\
\text { signal splitter }\end{array}$ \\
\hline $\begin{array}{l}\text { (2) Teledesign TS4000 radio } \\
\text { modems }\end{array}$ & Data cables: reciever-computer & $\begin{array}{l}\text { Tangent Mini S V2 } \\
\text { collection computer }\end{array}$ \\
\hline $\begin{array}{l}\text { TPL radio modem amplifier } \\
\text { LMR 400 cable: amp to large } \\
\text { base ant }\end{array}$ & & & Hypack survey software \\
\hline $\begin{array}{l}\text { (1) Laird large base antenna } \\
\text { (1) Laird small rover antenna }\end{array}$ & & & \\
\hline $\begin{array}{l}\text { Data cables: radio-receiver- } \\
\text { computer }\end{array}$ & & & \\
\hline \hline
\end{tabular}


Test Procedure and Data Collection. Test surveys were conducted on 1 August 2019. The objective was to evaluate the accuracies and performance of the three (RTK, RTN, RTX) GNSS correction systems during static and dynamic motion surveys. To ensure an accurate comparison of the three different systems on the rover, a single GNSS antenna was used with a three-way signal splitter. This use of a single antenna negated any potential data biases from multiple antenna offsets, inherent antenna signal differences, or other possible hardware differences.

The test consisted of nine independent survey logging sessions: six dynamic motion and three static motion. The dynamic motion logs were North Beach 1, North Beach 2, South Beach 1, South Beach 2, South Beach 3, and Dune. Each of these dynamic sessions was conducted using a John Deere Gator UTV (utility task vehicle) for the survey vehicle. The static motion logs were Static 1, Static 2, and Static 3. Each survey session began when all three systems were "Fixed" (meaning receivers were receiving and processing the corrections), and the logging session initiated. Hypack software was used to collect the survey data during the testing; positioning data of all three GNSS correction systems were received and recorded at $1 \mathrm{~Hz}^{1}$. All times are reported in Coordinated Universal Time.

Dynamic Surveys. North Beach 1 began inside FRF facility, transited to the beach, and drove north for approximately $10 \mathrm{~km}$ along the beach (Figure 1C, blue line). Heading was occasionally varied at random. After reaching the northern end point of the survey line, the UTV was then stopped, and the survey logging session ended.

North Beach 2 was the return trip back towards the facility. All systems were still online from previous session, and the survey logging session commenced. Course heading was again varied while transiting southward. Upon reaching the endpoint of the line, the UTV was stopped and the survey logging session ended.

South Beach 1 began at the FRF facility, transited to the beach, and then drove south for approximately $10 \mathrm{~km}$ along the beach (Figure $1 \mathrm{C}$, red line) while occasionally varying heading. After reaching the southern end point of the survey line, the UTV was stopped and the survey logging session ended.

South Beach 2 was the return trip back towards the facility. All systems were still online from the previous session, and the survey session was initiated. Course heading was varied at random. At the midpoint of the return trip, the UTV was stopped, and the survey logging session ended.

South Beach 3 began at the midpoint location were South Beach 2 ended. All systems were confirmed to be online prior to the start of a new survey logging session. The survey continued on the northward course while varying heading at random. Upon reaching the endpoint of the line, the survey logging session was ended.

Dune began at the FRF facility. All systems were confirmed to be online prior to initiating the survey logging session. The surveyed route started on the asphalt driveway then transitioned to

\footnotetext{
${ }^{1}$ For a full list of the spelled-out forms of the units of measure used in this document, please refer to US Government Publishing Office Style Manual, 31st ed. (Washington, DC: US Government Publishing Office 2016), 248-52, https://www.govinfo.gov/content/pkg/GPO-STYLEMANUAL-2016/pdf/GPO-STYLEMANUAL-2016.pdf.
} 
sand and vegetated paths through the dunes of the FRF northern property (Figure 1B, blue dotted line). Upon reaching the endpoint, the survey logging session was ended.

Static Surveys. All three of the static survey sessions were conducted at National Geodetic Survey (NGS) benchmark 1370D (Figure 1B, red circle); located near North Carolina Highway 12 , just to the north of the access road to the FRF facility. Static 1 began with the tripod centered over benchmark 1370D with center leg set to $1.5 \mathrm{~m}$ from the top centerpoint of the benchmark disc to the antenna reference point at the top of the tripod (Figure 2). Tripod legs were secured into the ground, and the tripod was leveled. The same GNSS antenna used during the dynamic sessions was secured to top of the tripod. All systems were confirmed to be online and Fixed. The survey logging session was conducted for $3 \mathrm{~min}$.

Static 2 began with no changes to the tripod setup. All systems were confirmed to be online and fixed; the survey session was conducted for $5 \mathrm{~min}$.

Static 3 began with no changes to the tripod setup. All systems were confirmed to be online and fixed; the survey session was conducted for $3 \mathrm{~min}$.

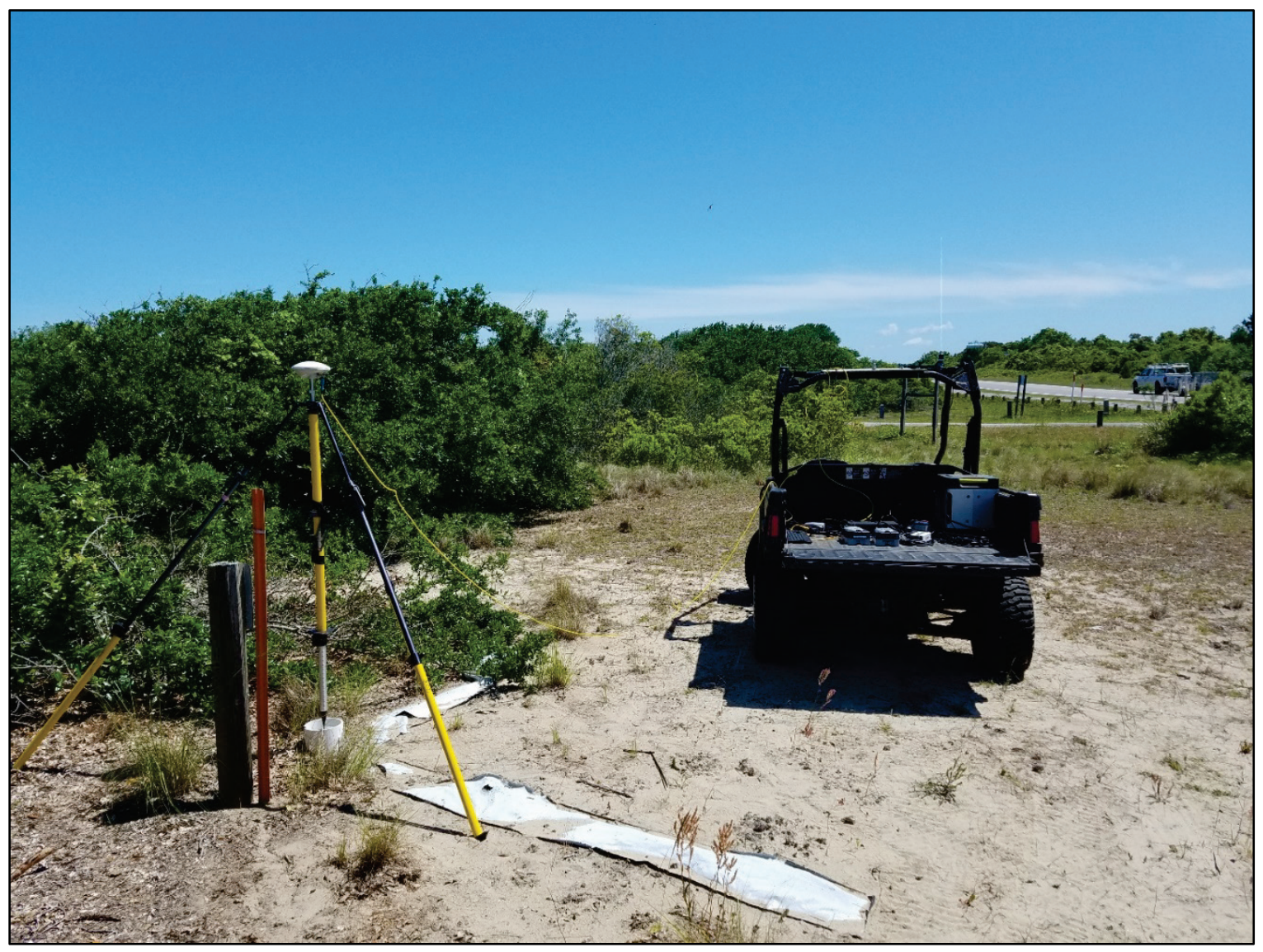

Figure 2. Static survey logging on NGS benchmark 1370D.

Data Processing. A Python script was developed to read in the raw data recorded in Hypack, time match the GPS data streams, identify and select only points coded "Fixed," and convert the geographic coordinates to North Carolina State Plane (Cartesian coordinates). A conversion from 
the International Reference Frame (2014) (ITRF) to the North American Datum of 1983 (NAD83) was also required for the RTX-based data. The RTX data stream provides corrections based on ITRF which references the World Geodetic System 1984 (WGS84) ellipsoid, while the RTN and Local RTK reference NAD83, which uses the Geodetic Reference System of 1980 ellipsoid. Errors between these two reference frames can be on the order of $0.50 \mathrm{~m}$ to $\sim 2 \mathrm{~m}$ in northeastern North Carolina. The National Geodetic Surveys Horizontal Time-Dependent Positioning (HTDP) online tool was used to convert between WGS84 and NAD83 datums (https://geodesy.noaa.gov/TOOLS/Htdp/Htdp.shtml). HTDP allows users to transform positional coordinates across time and between spatial reference frames.

\section{DATA ANALYSIS}

Static Observations. The static observations recorded at benchmark 1370D for the Local RTK, RTN, and RTX corrections methods were combined (three separate collects of instantaneous static data) and then subtracted from the NGS published coordinates. A series of boxplots (Figure 3) were created for X, Y, and Z values of each correction method. Box plots are a method for depicting groups of numerical data through a five-number summary (minimum, first quartile, median [depicted by horizontal line within box], third quartile, and maximum). The first quartile and third quartile are the lower and upper bounds of the box, respectively. The minimum and maximum are depicted by small horizontal lines at the end of the whiskers below and above the box. Outliers are displayed as dots above the maximum and/or below the minimum. An outlier is any value that lies more than 1.5 times the length of the box from either end of the box. The length of the box is also known as the Interquartile Range (also used as a measure of how spread out the values are) and is calculated by subtracting quartile 3 (top of box) by quartile 1 (bottom of box).

All correction methods had similar errors for horizontal coordinates $(\mathrm{X}, \mathrm{Y})$ with maximum differences of $+/-5 \mathrm{~cm}$ (Figure 3). These results are also consistent with the findings in Carballido et al. (2014) who evaluated error along pre-programmed lines for autonomous farm equipment using RTX positioning compared to a Local RTK system used to establish the tracklines. They reported root-mean-square-errors (RMSE) in X and $Y$ at $0.90 \mathrm{~cm}$ and $1.13 \mathrm{~cm}$, respectively, for RTX-corrected data while RMS errors were found in X and Y of $1.0 \mathrm{~cm}$ and $1.5 \mathrm{~cm}$, respectively. The Local RTK correction method showed the least amount of variance and outliers followed by RTN and then RTX. The Z elevation values showed the most spread with an RTX maximum error of $23 \mathrm{~cm}$. The outliers present in the RTX Z data are associated with the first data collect. Although the GPS receiver interpreted the solution as CenterPoint RTX, the elevations were consistently higher than the second two collects (Figure 4). After a discussion with the Trimble RTX technical support team, they confirmed that the quality indicator flag will only report if the solution is CenterPoint RTX, without indicating if the solution is converged/Fixed (converged represents best solution). The support team also suggested utilizing the GST (position error statistics) National Marine Electronic Association) message that reports the position error statistics of each record (NOT used during this test). By utilizing the GST message, a user could set tolerances based on statistics and likely eliminate or flag any suspect data. The authors suggested to Trimble RTX support team to add a flag indicator to CenterPoint RTX data to indicate if the solution is converged similar to the indicator flags for Float and Fixed RTK (float represents higher errors than fixed). 
Since the first data collect was likely NOT converged, the file was removed, and statistics recomputed to include only data where the solution had converged (Figure 5). When only considering converged solutions, RTX performed better with maximum differences in $\mathrm{Z}$ of $7 \mathrm{~cm}$ and a mean of $2 \mathrm{~cm}$.
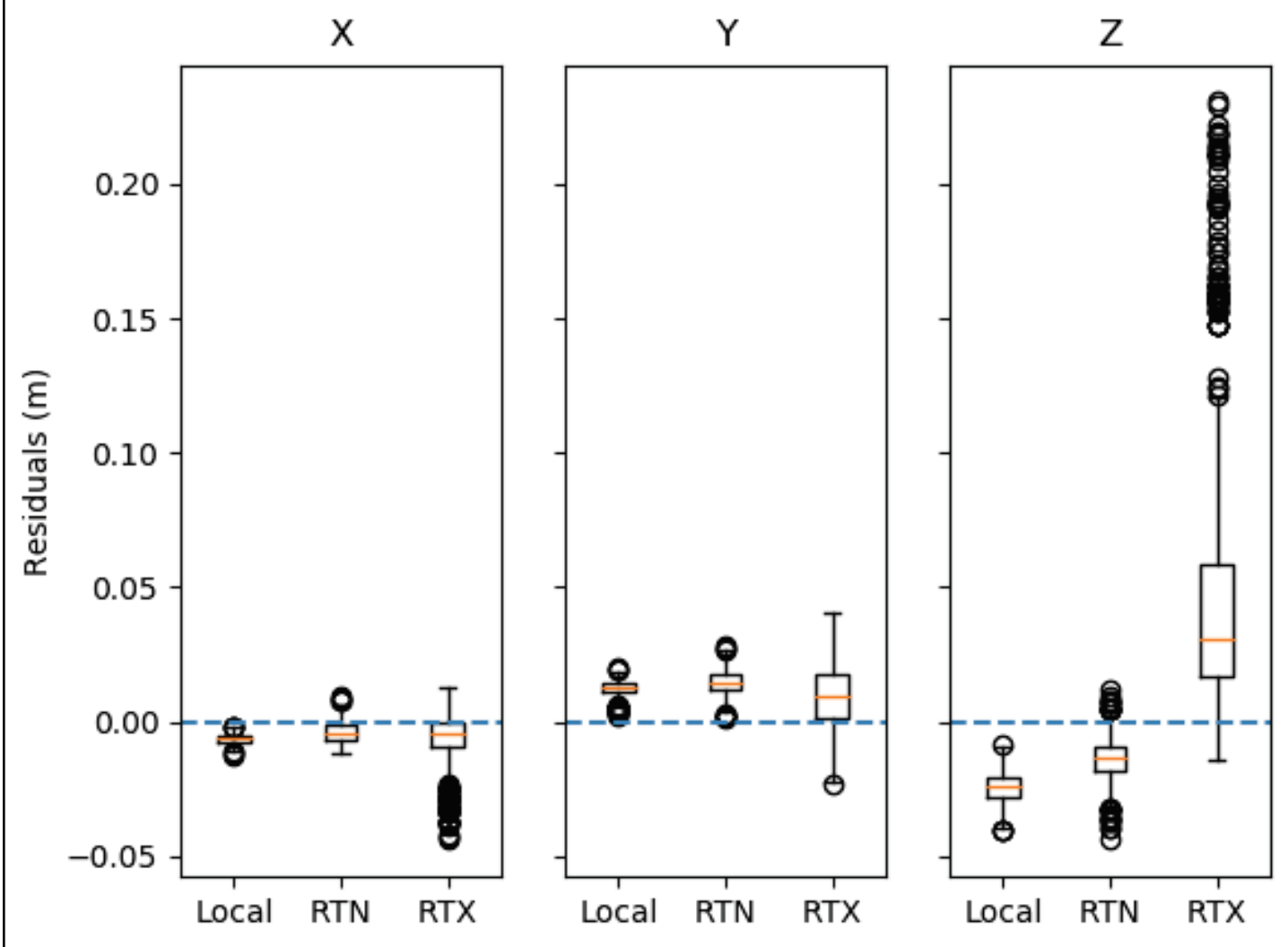

Figure 3. Static survey data boxplots displaying the distribution of data for each correction method and for each dimension $(X, Y, Z)$. Residuals are the differences between the observed data and the NGS published coordinates of benchmark 1370D. The benchmark is represented in the plots by the dashed blue line at 0.00 value. 


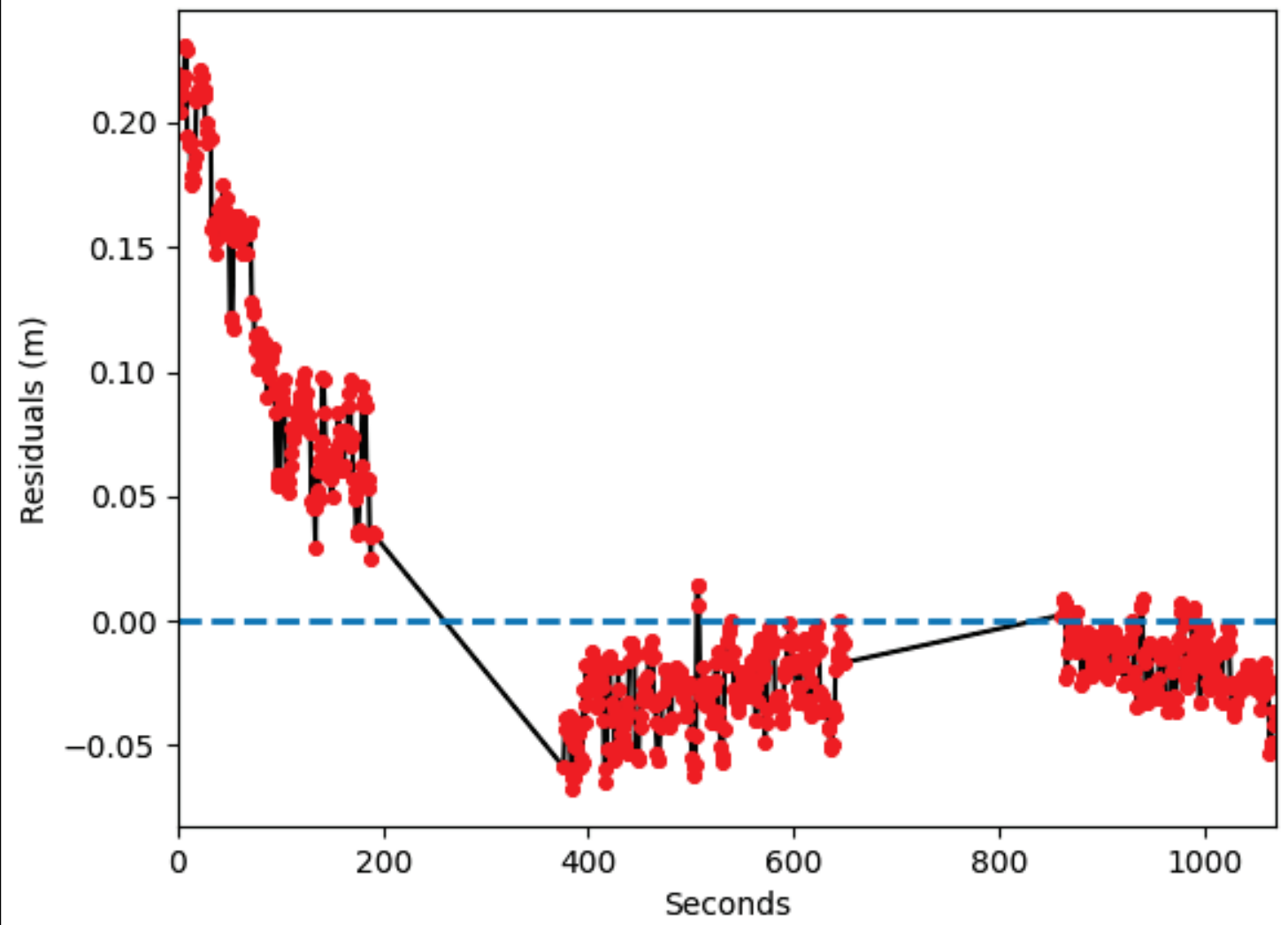

Figure 4. RTX elevation (Z) data: time series of static observations at NGS benchmark 1370 (three instantaneous data collects) demonstrating outliers at time $0-150 \mathrm{sec}$ likely representing a nonconverged Centerpoint RTX solution. The benchmark is represented by the dashed blue line at 0.00 value. Residuals are the differences between the observed data and the NGS published coordinates of benchmark 1370D. 


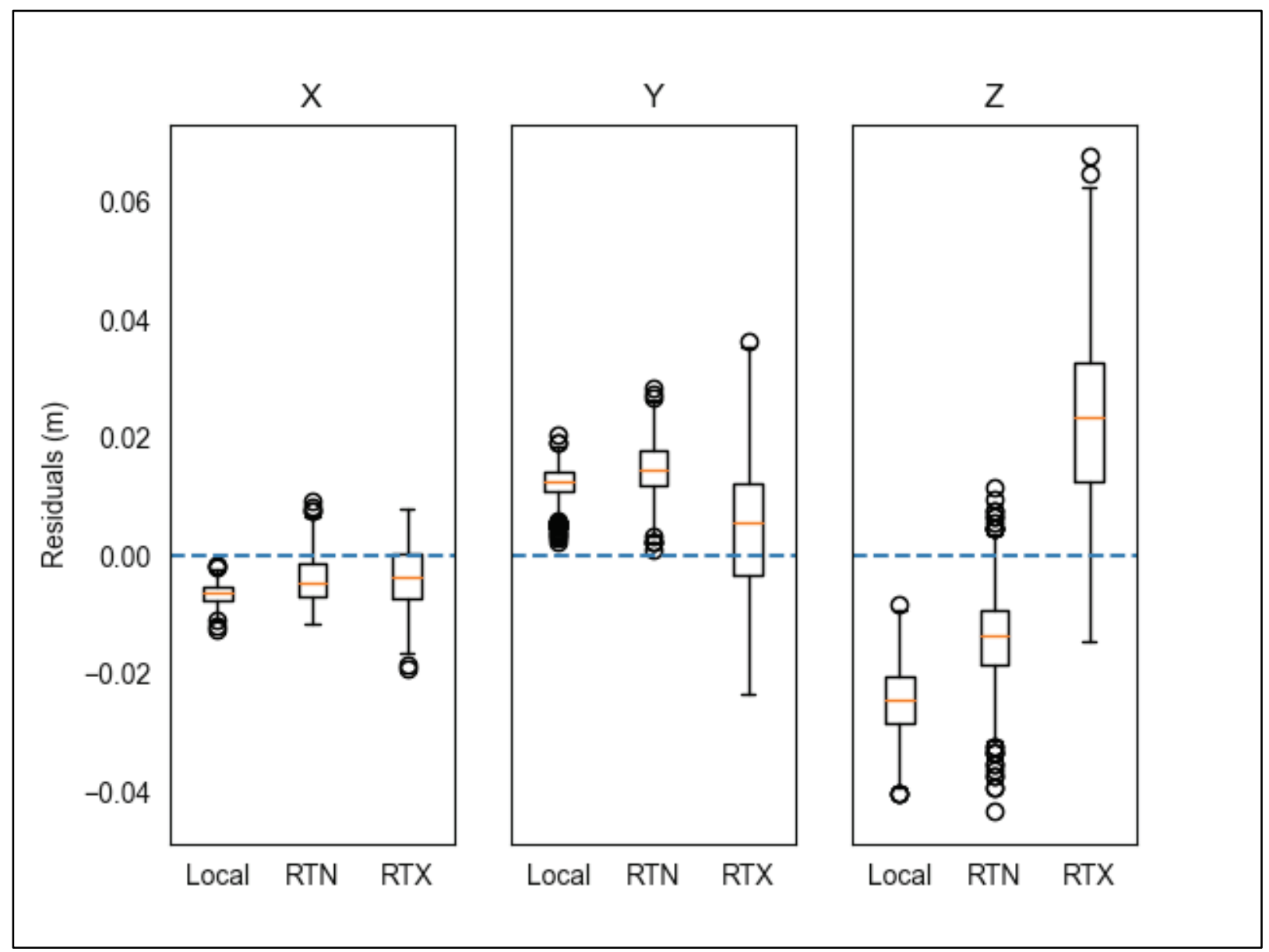

Figure 5. Boxplots displaying the distribution of data for each correction method and for each dimension $(X, Y, Z)$. RTX subset static survey data used for these plots with the nonconverged data removed. Residuals are the differences between the observed data and the NGS-published coordinates of benchmark 1370D. The benchmark is represented in the plots by the dashed blue line at 0.00 value.

The RMSE was calculated for each of the RTK correction methods using the following equation where $X_{o b s}$ is the RTK observations and $X_{\text {Groundtruth }}$ is the NGS benchmark and $n$ equal to number of observations.

$$
R M S E z=\sqrt{\frac{\sum_{i=1}^{n}\left(X_{\text {obs }, i}-X_{\text {Groundtruth }}\right)^{2}}{n}}
$$

The RMSE values were lowest for RTN followed by the Local RTK and then RTX. A negative bias was observed for both RTN and Local RTK while a positive bias was observed for the RTX. The large positive bias for RTX is attributed to the outliers that were observed during the first static logging session. The standard deviation was lowest for the Local RTK, suggesting a more stable solution. Increased errors have been shown in RMS Z $(6 \mathrm{~cm})$ for RTX corrections when compared to work by Leandro et al. (2011) who reported $2.8 \mathrm{~cm}$; however, when using the RTX subset (with non-converged data removed), a similar result of $2.3 \mathrm{~cm}$ is shown (Figure 6). 


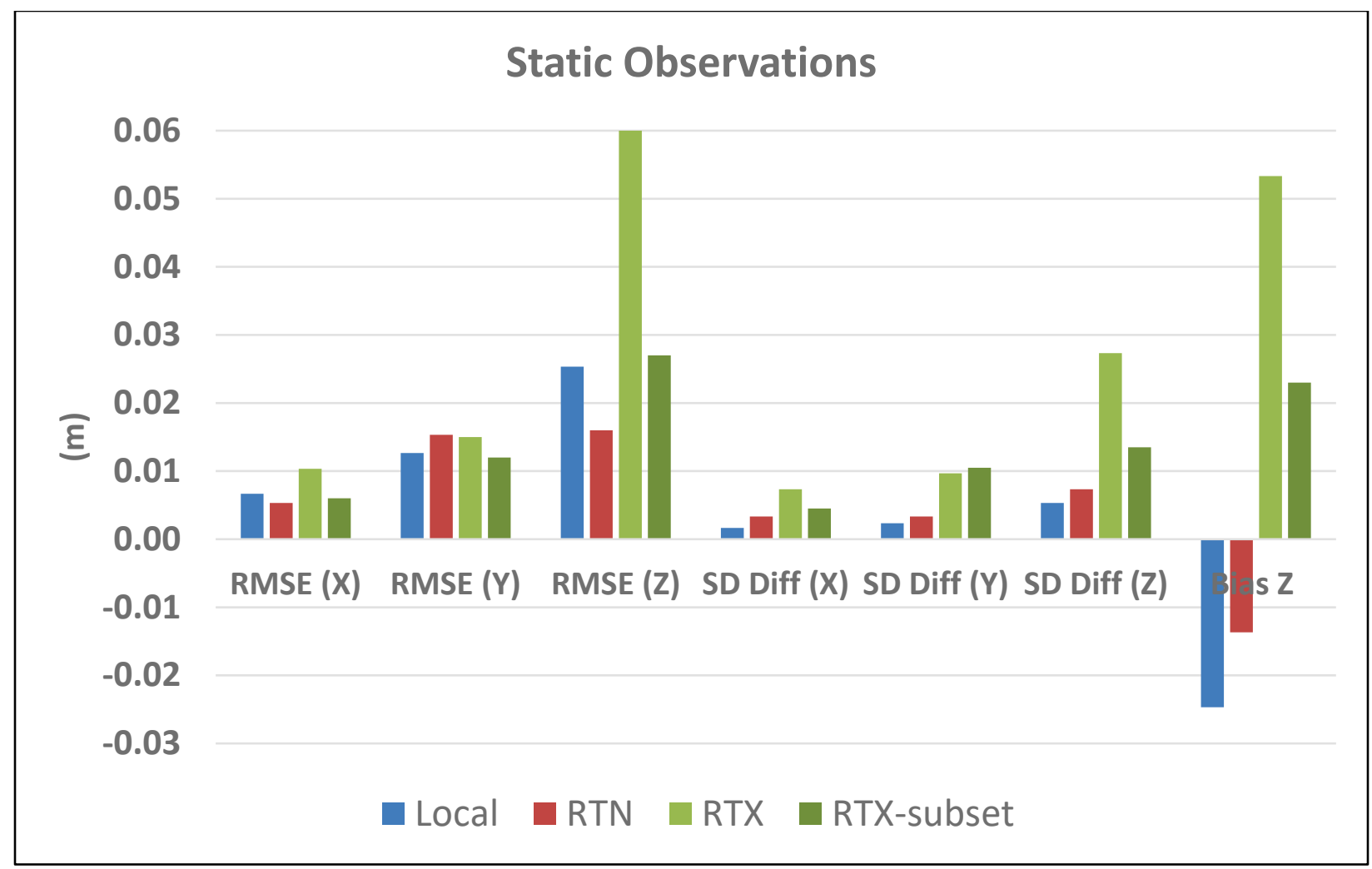

Figure 6. RMSE, Standard Deviation, and Bias (Z) for each of the RTK correction methods as they relate to the NGS-published coordinates for benchmark 1370D. The benchmark is represented in the plot as the 0.00 value on the $y$-axis. "SD Diff" is the standard deviation of the difference between RTN/RTX/Local values and benchmark 1370D values.

Dynamic Observations. Using the FRF Local RTK dynamic data as ground truth, the dynamic observations for both the RTX and RTN correction methods were subtracted from the Local RTK observations, and comparison statistics were developed. Although the local base station has associated errors, it is the method currently in operation for FRF survey collection and was used for the comparison. Under ideal satellite and atmospheric conditions, the horizontal accuracy of a Local RTK position is $\pm 1 \mathrm{~cm} \pm 1 \mathrm{ppm}$ based on the distance to the base station. Vertical accuracy is $\pm 2 \mathrm{~cm} \pm 2 \mathrm{ppm}$ (Trimble 2003). Since the maximum distance to the base station during the testing was $\sim 10 \mathrm{~km}$, the vertical and horizontal accuracy of the GPS positions was $\pm 4 \mathrm{~cm}$ and $\pm 2 \mathrm{~cm}$ respectively. The Local RTK also demonstrated the lowest standard deviations and tightest spread during the static observations. However, note that since both Local RTK and RTN had negative bias in Z in the static test, this comparison might favor RTN over RTX. The data for all the dynamic areas including the $20 \mathrm{~km}$ beach driving and the FRF property dunes were compiled, and box plots were created (Figure 7). 


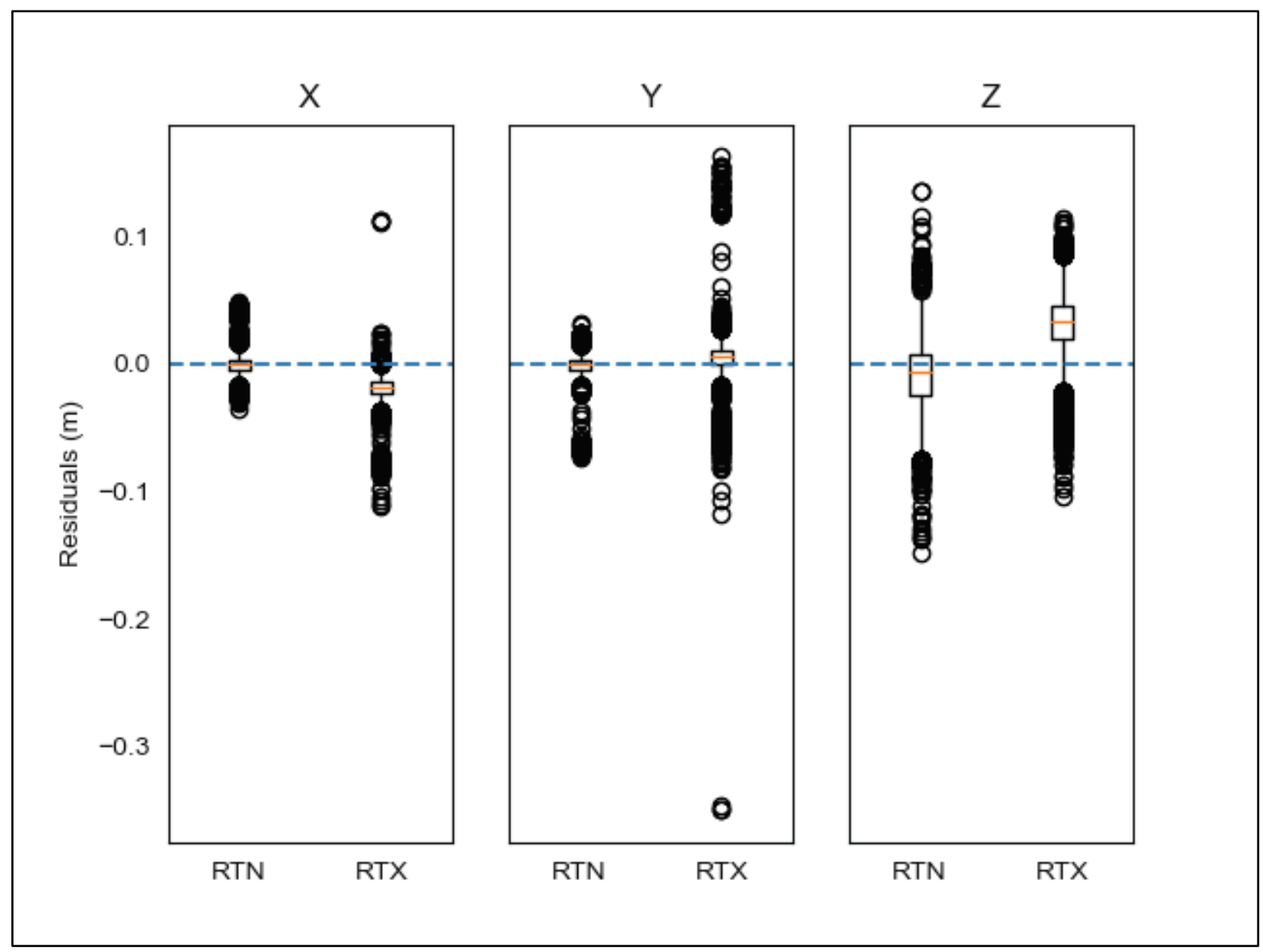

Figure 7. Dynamic survey data boxplots displaying the distribution of data for each correction method and for each dimension (X,Y,Z). Residuals are the differences between the observed Local RTK data and the observed RTN/RTX data. The Local RTK data are represented in the plots by the dashed blue line at 0.00 value.

RTN performed better than RTX when compared to Local RTK; however, both correction methods present outliers that should be noted. Of the 12,500 dynamic observations, the RTN X and Y data showed values $+/-5 \mathrm{~cm}$ while the RTX data were $+/-10 \mathrm{~cm}$ with a few outliers at $>30$ $\mathrm{cm}$. The $\mathrm{Z}$ values demonstrated more spread for both methods: RTN $+/-14 \mathrm{~cm}$ and RTX $+/-$ $11 \mathrm{~cm}$. Note that RTX dynamic observations did not contain any spurious outliers as observed in the static collects and likely represents a continuously converged solution.

Using the Local RTK data as the "ground truth," RMSE was calculated for the RTN and RTX correction methods using the former equation where $X_{o b s}$ is the RTN/RTX observations and $X_{\text {Groundruth }}$ is the Local RTK (Figure 8).

The RMSE values were lowest for the RTN observations across all three dimensions (X, Y, Z). Standard deviation was lower for the horizontal positioning of the RTN; however, the RTX data showed a slightly better standard deviation for Z. A negative bias was observed for the RTN while a larger positive bias was seen for the RTX data. The data also show lower RMSE in the horizontal (X, Y) for RTX corrections when compared to work by Leandro et al. (2011) who reported $2.3 \mathrm{~cm}$. 


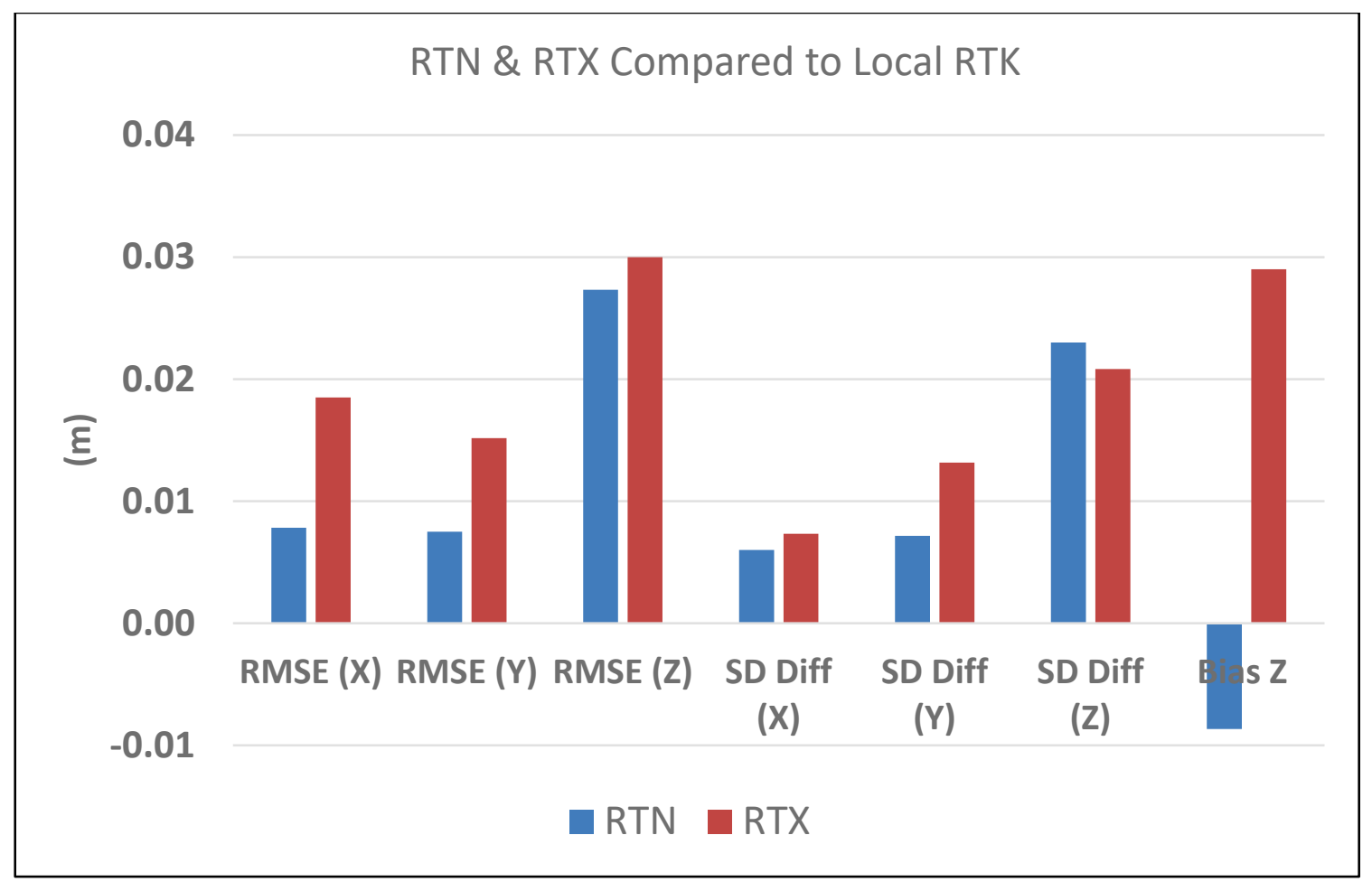

Figure 8. RMSE, Standard Deviation, and Bias (Z) for RTN and RTX correction methods as they relate to the Local RTK data. The Local RTK data are represented in the plot as the 0.00 value on the $y$-axis. "SD Diff" is the standard deviation of the difference between RTN/RTX values and Local RTK values.

CONCLUSIONS: Three GNSS correction methodologies (Local RTK, RTN, CenterPoint RTX) were evaluated to assess the performance in both static and dynamic environments. Although each methodology provides GNSS/GPS corrections to improve accuracy, they differ in terms of hardware, software, correction derived and delivery method, accuracy, and cost. The data from the dynamic testing showed that the RTN observations showed less error and differences vs. the RTX data when compared to ground-truth Local RTK data. In Z/elevation data, the RTX had lower standard deviation than RTN. In regard to the static data collections, both the RTN and Local RTK methods provided low RMSE values when compared to the local NGS benchmark. Both methods also had low standard deviations with Local RTK being slightly better, suggesting a more stable solution. In comparison, RTX reported the highest RMSE and standard deviations values. However, as previously mentioned, it is believed this is attributed to the first static data collect likely not being converged and had many outliers. When using the RTX subset data, the statistics were greatly improved and compared well with the other correction methods as all systems reported $<3 \mathrm{~cm} \mathrm{RMSE}$ in $\mathrm{X}, \mathrm{Y}$, and Z.

The three systems tested each have characteristics that users may find more or less appealing depending on their given positioning needs and work environment. The Local RTK system has the most user-owned and operated equipment needed to provide GNSS/GPS corrections and necessitates the set up of a base station (permanent or temporary). In addition, the Local RTK system uses radio transmission to deliver corrections from the base to the rover. There are pros and cons with both of these aspects of the Local RTK system, but given the results from this study as well as decades of use by numerous industries, the Local RTK system has proven a 
highly accurate and reliable system. The RTN system lessens the amount of equipment needed for GNSS/GPS corrections vs. the Local RTK system. However, RTNs may require access charges to use the service, and the work environments will need to have reliable and capable internet service to allow this correction method to function properly. The RTX system (satellite-delivered corrections) has the least amount of equipment required by the user, and the configuration of the RTX system allows this method to be used globally (except for high-latitude regions and more than $5 \mathrm{~km}$ offshore). Depending on accuracies required by the user, this can be advantageous to the user by negating the need of internet service (RTN) or the need to bring and set up all the equipment required for a Local RTK system.

ADDITIONAL INFORMATION: This CHETN was prepared by J. Robert Mitchell, John.R.Mitchell@usace.army.mil, and Michael F. Forte, Michael.F.Forte@usace.army.mil, US Army Engineer Research and Development Center, Coastal and Hydraulics Laboratory. Appreciation is expressed to Patrick J. Dickhudt and Ian W. Conery for insightful feedback on an early draft of this CHETN.

This CHETN should be cited as follows:

Mitchell, Robert J., and Michael F. Forte. 2021. Field Evaluation of GNSS/GPS Based RTK, RTN, and RTX Correction Systems. ERDC/CHL CHETN-IV-129. Vicksburg, MS: US Army Engineer Research and Development Center. http://dx.doi.org/10.21079/11681/41864

\section{REFERENCES}

Birkemeier, William A., and Michael F. Forte. 2019. Field Research Facility: A Guide to the Survey Lines Dataset. ERDC/CHL SR-19-5. Vicksburg, MS: US Army Engineer Research and Development Center.

Brandl, Markus, Xiaoming Chen, Herbert Landau, Carlos Rodriguez-Solano, and Ulrich Weinbach. 2019. “Anatomy of a Centimeter-Level Precise Point Positioning Service.” GPS World, May 12, 2019. https://www.gpsworld.com/anatomy-of-a-centimeter-level-precise-point-positioning-servicel.

Carballido, Jacob, Manuel Pérez-Ruiz, Luis Emmi, and Juan Agüera. 2014. "Comparison of Positional Accuracy Between RTK and RTX GNSS Based on the Autonomous Agricultural Vehicles Under Field Conditions." Applied Engineering in Agriculture 30(3): 361-366. https://doi.org/10.13031/aea.30.10342.

Forte, Michael F. William A. Birkemeier, and J. Robert Mitchell. 2017. Nearshore Survey System Evaluation. ERDC/CHL TR-17-19. Vicksburg, MS: US Army Engineer Research and Development Center.

Leandro, Rodrigo., Herbert Landau, Markus Nitschke, Markus Glocker, Stephan Seeger, Xiaoming Chen, Alois Deking, Mohamed BenTahar, Feipeng Zhang, Kendall Ferguson, Ralf Stolz, Nick Talbot, Gang Lu, Timo Allison, Markus Brandl, Victor Gomez, Wei Cao, and Adrian Kipka. 2011. "RTX Positioning: The Next Generation of cm-accurate Real-time GNSS Positioning." Proceedings of the 24th International Technical Meeting of the Satellite Division of The Institute of Navigation (ION GNSS 2011). Portland, OR.

Trimble. 2019. "Trimble RTX Frequently Asked Questions.” RTX FAQ Brochure. Accessed March 25, 2020. https://positioningservices.trimble.com/wp-content/uploads/2019/02/Trimble-RTX-FAQ-2020-Brochure.pdf.

Xiaoming, Chen, Timo Allison, Wei Cao, Kendall Ferguson, Simon Grünig, Victor Gomez, Adrian Kipka, Jan Köhler, Herbert Landau, Rodrigo Leandro, Gang Lu, Ralf Stolz, and Nick Talbot. 2011. "Trimble RTX, an Innovative New Approach for Network RTK." Proceedings of the 24th International Technical Meeting of the Satellite Division of The Institute of Navigation (ION GNSS 2011). Portland, OR. 
ERDC/CHL CHETN-IV-129

September 2021

NOTE: The contents of this technical note are not to be used for advertising, publication, or promotional purposes. Citation of trade names does not constitute an official endorsement or approval of the use of such products. 